\title{
Resisting motor mimicry: Control of imitation involves processes central to social cognition in patients with frontal and temporo-parietal lesions
}

\author{
Stephanie Spengler ${ }^{1}$, D. Yves von Cramon $^{2} \&$ Marcel Brass ${ }^{3}$ \\ ${ }^{1}$ Max Planck Institute for Human Cognitive and Brain Sciences, Leipzig, Germany \\ ${ }^{2}$ Max Planck Institute for Neurological Research, Cologne, Germany \\ 3 \\ Ghent University, Department of Experimental Psychology and Ghent Institute of Functional and \\ Metabolic Imaging, Ghent, Belgium
}

Running title: control of mimicry and social cognition

Keywords: perspective-taking, mentalizing, mirror system, shared representations, lesion study

Corresponding author:

Stephanie Spengler, Max Planck Institute for Human Cognitive and Brain Sciences, Department of Cognitive Neurology, Stephanstr.1a, 04103 Leipzig, Germany

e-mail: spengler@cbs.mpg.de

Tel: +49 (0) 34199402674

Fax: +49 (0) 34199402204

Acknowledgements:

This work was supported by the European Community's Sixth Framework Programme, NEST /EDICI (Contract No. 012929). 


\begin{abstract}
Perception and execution of actions share a common representational and neural substrate and thereby facilitate unintentional motor mimicry. Controlling automatic imitation is therefore a crucial requirement of such a 'shared representational' system. Based on previous findings from neuroimaging, we suggest that resisting motor mimicry recruits the same underlying computational mechanisms also involved in higher-level social cognitive processing, such as self-other differentiation and the representation of mental states. The aim of the present study was to investigate now on a behavioural level, whether there is a functional association between the inhibition of imitation and tasks, assessing the understanding of mental states and of different perspectives of self and other. In a sample of neuropsychological patients with frontal lesions, a correlation between the ability for mental state attribution and the control of imitation was found, with a similar effect in the control group. Temporo-parietal lesioned patients showed a highly significant correlation between imitative control and visual and cognitive perspective-taking. Even after controlling for executive functions, the results remained significant, indicating the functional specificity of this relationship. These findings provide new insight into the functional processes underlying the control of shared representations and suggest a novel link between embodied and higher-level social cognition.
\end{abstract}


Recent work based on the discovery of 'mirror neurons' has suggested that action observation is associated with the activation of an internal motor representation of this action, which can then be used to imitate the seen action (Iacoboni et al., 1999; Brass \& Heyes, 2005). This predicts an automatic tendency to imitate in the observer, corroborated by findings on unintentional imitation in social interaction ('chameleon effect', Chartrand \& Bargh, 1999) and a heightened display of imitative behavior in patients with prefrontal lesions (Lhermitte et al., 1986; Brass et al., 2003). Similarly, healthy participants exhibit prolonged reaction times and more errors when they had to execute an action, while viewing an incongruent movement at the same time (Brass et al., 2001a; Kilner et al., 2003). Due to these 'shared representations' for perception and action, a simulationist account has proposed that the mirror system is involved in action understanding, social cognition and imitation by motor simulation (Gallese \& Goldman, 1998). However, in healthy adults the potential to imitate is not always automatically translated into overt performance, thus raising the question by which cognitive and neural control mechanisms automatic motor mimicry is brought under intentional control.

One might consider two possible mechanisms being crucial for imitative control. Firstly, from an executive functions point of view, it could be plausible that imitative response tendencies do not differ from any other prepotent response tendency (e.g. overlearned responses as reading in the Stoop task), and that general cognitive control functions, such as response inhibition and interference control, are needed to inhibit unintended imitative behaviour. A second alternative is based on the assumption that a common representational system exists for actions, which are either externally perceived or internally planned ('common coding theory', Prinz, 1997), but that this system does not code explicitly by whom, oneself or another agent, the corresponding motor representation was evoked ('who' system, Jeannerod, 1999). This view would propose that the control of imitative behaviour requires mechanisms that enable a distinction between self or other related actions (Brass \& Spengler, 2008; Brass et al., 2009). This system first would need to distinguish between intended 
and externally triggered motor representations and, in case of a mismatch, reinforce representations, which were internally and intentionally generated.

Support for the latter view comes from recent neuroimaging studies, which used a paradigm specifically designed to investigate the inhibition of imitative behaviour (Brass et al., 2001b; Brass et al., 2005; Spengler et al., 2009). In this task participants had to lift their index or middle finger in response to a number while watching congruent (i.e. the same) or incongruent (i.e. the opposite) finger movements of a videotaped hand. As a first result, areas, which were involved in the inhibition of imitative behaviour, did not substantially overlap with areas involved in the inhibition of other prepotent response tendencies, measured by a version of the Stroop test, which was employed during the same fMRI session. This finding is mirrored by a lesion study showing that the performance in the imitation-inhibition task was not correlated with executive function measures, assessed by the Stroop task and the Wisconsin Card Sorting Test (Brass et al., 2003). Secondly, consistent with the hypothesis that the inhibition of imitative behaviour involves brain regions, which are related to selfother distinction and self-related, intentional processing, activations in the imitation-inhibition task were found in the medial prefrontal cortex (mPFC) and the temporo-parietal junction (TPJ). Neuroimaging studies have shown that these regions are necessary to distinguish self- and othergenerated actions and motor representations (Decety et al., 2002; Farrer \& Frith, 2002; Farrer et al., 2003; Leube et al., 2003), to shift one's cognitive (Ruby \& Decety, 2001, 2003) and visual perspective (Aichhorn et al., 2005), and to support mentalizing in 'theory of mind' (ToM) tasks (Gallagher et al., 2000). The median wall (including the precuneus) has also consistently been associated with tasks requiring self-referential processing (Northoff \& Bermpohl, 2004). Supporting this hypothesis, a recent neuroimaging study found considerable overlap of activations in mPFC and TPJ between the control of imitative behaviour and social cognitive functions, such as mentalizing, agency statements and self-referential processing (Spengler et al., 2009). This data thus suggests that 1) the inhibition of imitative behaviour is a special case of response inhibition, which does not 
primarily share neural regions and its implemented functional mechanisms with more general response inhibition mechanisms, and 2) the control of imitation draws on regions (mPFC / TPJ) and computational mechanisms implicated in higher-level social cognitive processing (e.g. Frith \& Frith, 2006a). This includes recognizing others' perspectives and separating the perspectives of self and others, an ability which is also required in spatial perspective-taking, and has been related to the TPJ. Moreover, it involves the building and maintaining of intentions and intentionality subserved by the mPFC. In the context of the imitation-inhibition task, but only in incongruent trials where the observed movement does not match the intended movement, these functions are needed to form and reinforce an internal intention against the imitative response tendency (mPFC) and to detect deviations of the observed behaviour from the motor intention (TPJ). Hence, both processes, the management and control of shared representations and the understanding of other people's mental states, require the ability to distinguish one's own mental representation from the other person's representation. We therefore assume that common functions underlie the control of imitation and tasks involving abilities such as mentalizing, perspective-taking (spatial as well as mental) or empathic responses. This would provide a novel link between two hitherto opposing theoretical views on social cognition (e.g. Keysers and Gazzola, 2007), by assuming that the control of mirror system functions ('simulation account') relies on key computations of mentalizing and self-other distinction ('theory of mind account').

The present study was designed to investigate the relationship between the inhibition of automatic, motor mimicry and tasks involving aspects of higher-level social cognitive processing, such as perspective-taking, in the visual and cognitive domain, second-order representation of mental states and agency processing. As previously acquired information about the neural correlates on the inhibition of imitation was used to derive our hypotheses (Spengler et al., 2009), we sought to combine these neuroimaging results with behavioural data from a lesion study. Neuropsychological studies often yield additional or sometimes even different results than fMRI studies and can also 
provide further information about the necessity of sub-processes or areas for a particular cognitive function (e.g. Baird et al., 2006; Fellows \& Farah, 2005; Swick \& Turken, 2002). To address the first aim of the study, we used a correlational approach to investigate whether the control of imitation is functionally associated with aspects of social cognition. A group of neuropsychological patients with lesions either in the frontal cortex or around the temporo-parietal junction were investigated. Both regions have been found to be involved in the attribution of mental states, as well as during self-other distinction, and perspective taking. The TPJ was found to be involved not only in mental, but also spatial perspective-taking (e.g. Aichhorn et al., 2005). Patients thus completed the imitationinhibition task, a ToM task, empathy questionnaires (including a scale on cognitive perspectivetaking) and a visual perspective-taking paradigm. Specifically, we predicted for the frontal group that impaired performance in the imitation-inhibition task should be associated with impaired performance in the ToM task. For the temporo-parietal group, it was expected that deficits in the control of imitative behaviour should also be accompanied by decreased performance in mentalizing, as well as spatial and cognitive perspective-taking. Furthermore, the influence of possible moderating variables on these correlations was examined to ascertain their impact on the correlations and to elucidate the role of executive functions.

\section{Methods}

\section{Participants}

A total of 28 patients with acquired focal lesions of various etiologies were included in the study (see Appendix). Etiologies included traumatic brain injury (TBI), tumors, cerebrovascular etiology and infections. Patients were divided in a group with frontal lesions $(n=15)$ and a group of patients with lesions around the temporal-parietal junction (TPJ) $(n=13)$. The two patient groups were recruited from a database of the Day Clinic of Cognitive Neurology, which they had attended for rehabilitation purposes. They were assigned to the groups by an experienced neurologist (D. Y. 
C.) according to their MRI/ CT scan. For the frontal group, lesions were included if a selective lesion in the frontal cortex was present (either lateral or medial part), but not in the temporal or parietal cortex. Some lesions also included the basal ganglia and the insula. In the temporo-parietal group, lesions had to include primarily the TPJ region, but no lesions should be present in the frontal cortex. Further, only patients with an age between 18 and 70 years were included, and participants with sedative medication and a history of alcohol or drug abuse were excluded. Based on a prior neuropsychological examination patients were included in the study if they had no severe neuropsychological deficits (aphasia, neglect, amnesia, agnosia, severe attentional or motor deficits) and possessed the ability to understand the instructions. At the time of testing all patients were at least 6 months post injury (mean 6.2 years, $S D=4.4$ years) to ensure that all patients were in a chronic stage, where no extreme changes in performance can be expected. The control group was individually matched (on the basis of age, sex, education) to the patient groups $(n=28)$ and had no history of neurological or psychiatric diseases. They were recruited from a database at the Max Planck Institute. Demographic characteristics of the three groups are shown in the in Table 1. No significant differences were detected between the three groups for age, sex, education level, and premorbid IQ (one-way ANOVA, for all comparisons $\mathrm{p}>0.4$ ), and gender $\left(\mathrm{chi}^{2}\right.$ test, $\mathrm{p}>0.5$ ). From the control group one participant was not included in the sample as his error rate in the visual perspective-taking task was five standard deviations above the mean of the control group. In the patient sample, all participants completed the imitation-inhibition task, the Theory of Mind task and the questionnaires. For the remaining tests three frontal patients refused to take part in some of these tests, including the Stroop test, the visual perspective-taking task and the Wisconsin Card Sorting Test. Three participants from the temporo-parietal lesion group were not able to perform the visual perspectivetaking task and two did not perform the Stroop task. The study was approved by the University of Leipzig local ethical committee and all participants gave their informed consent in compliance with the Declaration of Helsinki. 


\section{Materials and Procedure}

All participants were tested in an individual session lasting approximately 2 hours at the Max Planck Institute. In the first part of the session all experimental tests (imitation-inhibition task, ToM test, empathy questionnaires, visual perspective-taking task) were administered in a fixed order to ensure that participants completed all experimental tasks before several neuropsychological measures on executive functions and intellectual functioning were administered. This included a computerised, manual version of the Stroop task to test for deficits in response inhibition and interference control and the Digit Span subtest (forward and backward) from the revised Wechsler Memory Scale (WMS-R, Wechsler, 1987) to assess working memory capacity. Premorbid, verbal intelligence was estimated with the Mehrfachwortschatz Test (MWT, Lehrl, 1999), a German equivalent of the National Adult Reading Test (Nelson \& Willison, 1991). Nonverbal, fluid intelligence was obtained with a subscale (LPS-3) from a German intelligence scale (Leistungsprüfsystem, Horn, 1983), which was designed to measure logical reasoning. Basic neuropsychological data was available for most patients on further executive functions tests. This included the Wisconsin Card Sorting Test (Grant \& Berg, 1948) or its modified version (Nelson, 1976) assessing mental flexibility and response inhibition (only for the frontal lesion group). When designing the study it was deliberately chosen not to re-administer the WCST, as in previous studies the test-retest reliability of the WCST yielded low coefficients (e.g. Bowden et al., 1998).

Imitation-inhibition task. In this task (Brass et al., 2000, 2003, 2005) participants had to lift their index or middle finger in response to a number (1: index finger, 2: middle finger), while watching video sequences of a hand, which mirrored the hand of the subjects. The task consisted of three different conditions (Fig. 1). In the baseline condition only the number was presented while the hand on the screen remained motionless. In the congruent condition the corresponding finger on the screen was lifted simultaneously with presentation of the digit (e.g., the index finger was lifted when 1 was presented). In the incongruent condition the noncorresponding finger was lifted (e.g., the 
middle finger was lifted when 1 was presented). Every video sequences started with a frame showing the hand in a resting position $(2000 \mathrm{~ms})$, two consecutive frames (each lasting $34 \mathrm{~ms}$ ) with the number and the finger movement, finishing with a frame $(1240 \mathrm{~ms})$ showing the finger in the end position. During trials a blank screen was presented for $2000 \mathrm{~ms}$. Reaction times were recorded with a custom-built response device, which used light sensors to detect finger lifting movements. The imitation-inhibition task started with a 20-trial practice phase. The experimental part consisted of three 50-trial blocks with short breaks between them and the conditions were presented randomly.

Advanced Theory of Mind task: 'strange stories'. In this task mental state understanding is tested with 16 short stories, half of them requiring the comprehension of a mental state and the other half of a physical state (Happe et al., 1999). The stories include themes of double bluff, white lie, persuasion or being tactful. Each passage was read aloud to the participants while a copy of the story was placed in front of them to read the story themselves. This procedure was chosen to account for reading or visual problems of some patients and to assure they understood the story. Participants were encouraged to absorb as much information as possible, as they were not allowed to refer back to the passage once they had seen the question. After the participants felt they remembered the details and understood the story the sheet was turned over to reveal a question. This question asked about the protagonists' thoughts and feelings and remained in front of the subjects during their response. Responses were recorded, transcribed and rated by two independent raters according to the standardised rating scheme developed by Happe et al. (1999). Answers were scored with two points if they gave a full and explicit account, one point for an implicit or partial response and no points for incorrect answers (examples of the stories and the rating criteria can be found in Happe et al., 1999). In addition, the number of verbs referring to a mental state (e.g. think, know) were counted. Interrater-reliability was high between the first author and a co-rater blind to group and all aspects of the experiment (intraclass correlation coefficient $=0.89$ ). 
Empathy questionnaires. Two different self-report questionnaires were used to assess empathy and its key sub-components, such as perspective-taking abilities. The Interpersonal Reactivity Index (IRI, Davis, 1983) was chosen to assess cognitive and affective components of empathic ability, as empathy has been considered as a multidimensional construct by different researchers (e.g. Davis, 1983). The scale consists of four seven item subscales. These assess cognitive perspective-taking and fantasy, representing the cognitive aspects of . It also includes an empathic concern subscale, tapping affective facets of empathy. A fourth scale measuring personal distress was not analysed further, as it is not always considered as a core component of empathy and was found to be negatively correlated with perspective-taking (Davis, 1980). A German version of this questionnaire was used (Saarbrückener Personlichkeitsfragebogen, Paulus, 2004), yielding high reliability coefficients, which were comparable to the original english version. To ensure that the participants understood the items and the rating scale, all items including a negation were rephrased with simpler sentences with no negative formulation, as a pilot study showed that patients had problems to comprehend the more complicated negation. In addition, each item was read out to the participants and help was provided if problems occurred with the rating scale. Furthermore, a subscale measuring ideomotor empathy was used (Enz et al., 2004). This 8-item scale assesses, additionally to cognitive aspects of empathy (“As a child I was good at playing scissor, stone, paper.", "I am good at predicting peoples' behaviour."), also ideomotor aspects of empathy ("When I see someone dancing, I also want to dance”.). Unfortunately, three participants were not familiar with the 'paper, scissor, stone game', therefore could not provide an answer to this questions and were excluded in the analysis.

Visual perspective-taking task. To test the ability for visuo-spatial perspective-taking, participants had to perform a modified version of a task, which required a egocentric perspective transformation (Zacks et al., 2002), that is an imagined change of one's own orientation and position to map one's spatial frame of reference onto that of another person. In this computerized task participants had to make judgements about colour drawings of a human body with each picture 
showing a figure facing the viewer and outstretching one arm (either away from the body or folded over the chest). The orientation of the body could vary in 30 degree steps from 0 to 180 degrees (clockwise or anti-clockwise from upright) while each of the seven possible orientations occurred equally often. Participants had to perform two tasks in response to these stimuli, which were blocked (a pilot study showed that switching between task on each trial was found too difficult), and their order was counterbalanced. In the control task (side condition) subjects had to judge on which side of the monitor the outstretched arm occurred (from their point of view) and respond by pressing a button either with the right of left index finger. This task was chosen to control for perception of human bodies and left-right decisions. In the experimental condition (hand condition) participants reported whether the body's left or right arm was extended from the figure's point of view with a left or right button press, respectively. In each trial the stimulus was displayed for maximal duration of 3000ms. During trials a blank screen was presented for $1200 \mathrm{~ms}$. In total 112 trials were presented for each condition divided into two blocks. For the arm task all possible combinations of pose, extended arm and orientation were tested twice and, in order to also obtain equal numbers of the seven orientations, pictures with orientations of 0 and 180 degrees were tested twice. For the side task only orientations, which unambiguously allowed a classification of the arm being on the left or right side of the monitor were used. Twelve practice trials were presented for each of the conditions. Previous studies have shown that the presentation of bodies with a spatial judgement relative to the pictured body spontaneously elicited imagined egocentric perspective-transformations of the viewer to solve the task, indexed by behavioural data and introspective reports (Parsons, 1987; Zacks \& Tversky, 2005). Additionally, it was chosen to instruct the participants explicitly to perform such a spatial perspective transformation. Before the experimental condition participants were instructed to answer the questions by "imaging yourself in the position of the figure on the screen" and to clarify the instructions participants had to physically demonstrate the imagined transformation to be performed with two test trials. 
Stroop task. A computerized, manual version of the Stroop task was used to assess the inhibition of overlearned response tendencies (Brass et al., 2003, 2005). This version allowed us to assess reaction times in addition to errors and to use a version of the Stroop task which was similar to the imitation-inhibition task. Stimuli in the colours red, green, blue, or yellow were presented in the middle of the computer screen. In the baseline condition XXXX was presented on the screen coloured in one of the four possible colours. In the congruent condition color words were presented in their matching color (e.g., RED in red). In the incongruent condition colour words were presented in the nonmatching colour (e.g., RED in blue). Thus, there were four possible response alternatives and four response buttons corresponded to one of the colours used in this task. Participants had to respond by pressing the response button which matched the colour of the stimulus presented with their middle or index finger of the left or right hand. To minimize working memory load, we presented a two-letter abbreviation of the colour words in the position of the respective response button below the stimuli. Each trial started with the presentation a blank screen for $1000 \mathrm{~ms}$. After presentation of a fixation cross for $200 \mathrm{~ms}$, the item and the abbreviations of the colours were presented for a maximal duration of $3000 \mathrm{~ms}$. Afterwards a short feedback on the correctness of the response was displayed on the screen for $500 \mathrm{~ms}$. The Stroop task started with a 20 -trial practice phase. Afterwards three blocks of 36 experimental trials followed.

\section{Statistical Analysis}

All statistical analyses were performed with SPSS (Version 15.0). None of the variables, except for errors in the Stroop task, showed a significant difference from a normal distribution using the one- sample Kolmogorov-Smirnoff test $(\mathrm{p}>0.05)$. As small sample sizes $(\mathrm{N}=10-15)$ in the patient groups were obtained, non-parametric tests were used, if not other specified. Furthermore, the majority of the dependent variables had an ordinal metric scale (interval data: e.g. reaction times, errors; ordinal data: e.g. variables with Likert scale items). Thus, correlations between the imitationinhibition task and the experimental tasks (ToM task, empathy questionnaires, visual perspective- 
taking task) were computed using Spearman's rho correlations. A Bonferroni correction ${ }^{1}$ was applied to adjust the significance level (one-tailed for the main comparisons (imitation-inhibition task \& social cognitive tasks) with prediction), to account for multiple comparisons (i.e. correlations) between the dependent variables of imitation-inhibition task and each of the experimental tests, separately for each group. To investigate the influence of possible confounding variables on these correlations, partial correlations (uncorrected, one-tailed), as implemented in SPSS, were computed. All other, not predicted correlations were two-tailed and uncorrected. Raw scores were used for the analysis, except for the two IQ measures (MWT, LPS 3), where IQ scores were used, and the Wisconsin Card Sorting Test, or its modified version, using percentiles. Due to small group numbers no subgroups (e.g. lesion side) were analyzed within the patient groups.

\section{Results}

\section{Correlations between the Imitation-Inhibition Task and the Social Cognitive Tasks}

To test the hypothesis of a functional relationship between the inhibition of imitation and social cognitive abilities, correlations between those variables, for each of the main groups (frontal lesion patients, temporo-parietal patients, control group), were computed. It was hypothesised that decreased performance in the imitation-inhibition task, indicated by a higher interference score (in reaction times or errors), should be accompanied by impaired performance on the other tasks, for example more errors or lower scores. Therefore, negative correlations were expected for variables, where lower performance would lead to lower scores (ToM task, empathy questionnaires), but positive correlations for variables, which indicated decreased performance by higher values (e.g. errors or RTs in the visual perspective-taking task).

Theory of Mind. In the frontal lesion group a highly significant correlation $(r(13)=-.75, \mathrm{p}<$ 0.01, Bonferroni corrected, effect size: Cohen's $d=2.2$ ) between the imitation-inhibition task and the accuracy score in the mentalizing condition of the advanced Theory of Mind task was found (Table 
2, Fig. 2, top row). Patients with a high interference score displayed decreased performance on the ToM task. Confirming this result, the second dependent variable of the ToM task, number of used mental state verbs, correlated significantly with the interference score (RTs) of the imitationinhibition task as well $(r(13)=-.62, \mathrm{p}<0.05, \mathrm{~d}=1.5)$. The control condition (physical stories) showed a trend to a significant correlation with the imitation-inhibition task, but failed to reach significance on the corrected alpha level. No significant correlations could be found in the temporoparietal lesion group in the ToM task. Like the frontal group, participants of the control group showed a significant correlation between the imitation-inhibition task (RTs interference score) and the accuracy score of the ToM condition as well $(r(25)=-.38, \mathrm{p}<0.05$, uncorrected for multiple comparisons, $d=0.8)$. However, this correlation was smaller than for the frontal group $(\mathrm{p}<0.1)$, and the correlation in the frontal group was significantly larger than in the temporo-parietal group (p < $0.01)$.

Empathy. Correlations between the subscales of the empathy questionnaires and the imitationinhibition task showed a highly significant, negative relationship in the temporo-parietal lesion group (Table 3). Patients with lesions around the TPJ area with a high interference effect in the imitationinhibition task displayed lower scores on the cognitive perspective-taking scale of the IRI (Fig. 2, bottom row) and the ideomotor empathy scale $(r(11)=-.77, \mathrm{p}<0.01$, corrected, $\mathrm{d}=2.4 ; r(8)=-.74$, statistical trend, $d=2.2$ ). No significant correlations could be found between the imitation-inhibition task and the different subscales of the empathy questionnaires in the frontal group and the control group (range $r(26)=-.01-.19, \mathrm{p}>0.31$, uncorrected) and the correlation in the cognitive scale was significantly smaller than in the temporo-parietal group (both groups $\mathrm{p}<0.01$ ).

Visual perspective-taking. Considering the third experimental task on visual perspectivetaking, a significant positive correlation $(r(8)=.75, \mathrm{p}<0.05$, after Bonferroni correction, $\mathrm{d}=2.2)$ emerged between errors (including omissions) in the hand condition of this task and the interference score (RTs) in the temporo-parietal group (Table 4, Fig. 2, bottom row). The direction of this 
correlation was again predicted by the hypotheses and was specific to the hand condition. To rule out the possibility that this correlation was just due to possible, underlying spatial compatibility effects in both tasks, the trials of the hand condition in the visual perspective-taking tasks were divided into spatial compatible (correspondence between correct response hand and monitor side of the figure's arm), incompatible (difference between correct response hand and monitor side of the figure's arm) and neutral trials (arm of the figure is in the midline of the monitor). Contradicting a spatial compatibility interpretation of the results, interference effects (RT) in the imitation-inhibition task were significantly correlated with errors in neutral and compatible trials $(r(8)=.68, \mathrm{p}<.05$, uncorr. $)$, but not in incompatible trials $(r(8)=.55, \mathrm{p}>0.1$, uncorr.). This result suggests that functions other than spatial compatibility must be shared between these two tasks. No correlations were found between performance in the visual perspective-taking task and the imitation-inhibition task in the frontal lesion group $($ range $r(11)=.00-.21, \mathrm{p}>0.42$, uncorr. $)$, or in the control group (range $r(26)=$ $.01-.28, \mathrm{p}>0.14$, uncorr.). The correlation of the temporo-parietal group was significantly larger than in the frontal group $(\mathrm{p}<0.01)$, and showed a trend towards statistical significance for the control group $(\mathrm{p}<0.1)$. Interestingly, visual perspective-taking, measured by errors in the hand condition, was also significantly correlated with self-reported cognitive perspective-taking abilities $(r$ $(8)=.881, \mathrm{p}<0.001$, uncorr., $\mathrm{d}=3.7)$ in the temporo-parietal group; no other correlations could be found between this task and the other experimental variables ( $\mathrm{p}>0.05$, uncorr).

\section{Additional Analyses}

The data clearly support the hypothesis of a functional relationship between the imitationinhibition task and the experimental measures. However, it is important to ascertain that these bivariate correlations are not just due to outliers, which may artificially inflate a correlation, especially in small groups and in special populations. Two commonly used procedures (Cook's Distance metric and leverage values) were used to test the assumption whether correlations were influenced disproportionately by individual subjects. Conventionally, it is stated that for Cook's 
Distance metric values $>1$ should be considered as statistical outliers, whereas for the leverage statistic 0.5 is taken as the cutoff. In the visual-perspective taking task only one participant in the temporo-parietal group and one participant in the control group showed higher values and were excluded from the analysis in this task. Further, one participant of the control group show a higher value for the partial correlation of the ToM task (see below) and was therefore excluded. None of the data points of the remaining, above-reported correlations between the imitation-inhibition task and the experimental task showed a value greater than 0.34 (for both measures) and most data points had values smaller than 0.1 . Therefore, the above-reported, significant correlations are not caused by statistical outliers.Furthermore, as one rationale for the use of brain-damaged patients was due to the assumed, higher variance of this group, the variances of the patient and control group were compared. Confirming this idea, in all task patients showed a higher variance and, most crucially, in the two dependent variables of the imitation-inhibition task this difference was significant (Levene's test for equality of variances, $\mathrm{p}<0.05)$.

\section{Influence of Possible Moderating Variables on Correlations}

To assess the possible moderating effect of additional variables on the foregoing, significant correlations between the imitation-inhibition task and social cognitive and visual perspective-taking tasks, partial correlations with different variables, were computed.

Firstly, demographic variables (age, education), premorbid IQ/ fluid intelligence and time since lesion were controlled for in a partial correlation between the variable of the imitationinhibition task and the dependent variables of the experimental tasks, which already yielded significant correlations as reported in the last paragraph. All correlations remained significant $(\mathrm{p}<$ .05 , uncorr.) after controlling for those variables.

Secondly, it seemed important to control also for the performance of the control condition (physical stories) of the ToM task, when looking at the correlation with the imitation-inhibition task in the frontal group. Even after entering the physical condition as a control variable in a partial 
correlation the relationship between the ToM condition and the interference score was still significant $(r(13)=-.605, \mathrm{p}<0.05$, uncorr., $\mathrm{d}=1.5)$. This finding was replicated in the control group and the correlation even increased after accounting for the physical control condition $(r(25)=-.475, \mathrm{p}<0.01$, uncorr., $d=1.0$ ). Figure 3 illustrates this relationship and provides evidence for our idea, that subprocesses, attributable to mentalizing abilities, are shared with the ability to control automatic imitation.

In a third step the effect of tasks measuring different aspects of executive functions, namely cognitive flexibility (WCST), working memory (backwards span) and interference control (Stroop), were investigated. Consistent with a previous study with frontal lesion patients (Brass et al., 2003), no significant correlations could be found in the frontal group between the variables of the imitationinhibition task and the variables of the WCST/ MCST (categories, errors, perseverative errors, all p > 0.50, uncorr.). For the both patient groups no significant correlations emerged with the Stroop task (frontal: $\mathrm{p}>0.14$, uncorr.; temporal-parietal: $\mathrm{p}>0.53$, uncorr.) and the backwards span (frontal: $\mathrm{p}>$ 0.50, uncorr.; temporal-parietal: $\mathrm{p}>0.86$, uncorr.). When controlling for the performance of these tasks in a partial correlation all above reported correlations between the imitation-inhibition task and the different experimental variables remained significant ( $\mathrm{p}<0.05$, uncorr.). Thus, the main correlations revealed in the last paragraph are not due to the influence of the moderator variables, which were considered in this study. The results therefore strongly support the hypothesis, that uniquely shared functions between the control of imitation and key processes implicated in social cognition exist.

\section{Discussion}

Recent evidence from neuroimaging studies showed that the inhibition of imitative behaviour and social cognitive abilities yielded overlapping activation in the medial prefrontal lobes and the temporo-parietal junction. Based on these findings we hypothesized that higher order social cognitive abilities, such as mentalizing, self-referential processing and awareness of agency, are 
based on basic functional mechanisms that are also needed to distinguish competing motor representations. The aim of the present study was to test this functional relationship in patients with frontal and temporo-parietal lesions. Our results strongly indicate that the ability to inhibit automatic imitative responses is associated with mentalizing performance and with the ability to identify different perspectives in the spatial and mental domain. As predicted, our data additionally show a differential correlational pattern for the frontal and temporo-parietal lesion group, respectively. Furthermore, a similar correlational pattern was also observed in healthy adults. Moreover, these correlative patterns could not be explained by a broad range of possible moderator variables, including also executive functions.

\section{Functional Associations between Imitative Control and Social Cognition}

Evidence from recent neuroimaging studies on the control of imitative behaviour and other studies on social cognition show that very similar activation foci of those two concepts were evoked in the mPFC and the TPJ (Brass et al., 2005; Decety \& Lamm, 2007; Gilbert et al., 2006; Spengler et al., 2009), supporting the sharing of functional mechanisms. It was therefore hypothesized that functional mechanisms needed to distinguish competing motor representations of actions are also required in higher-level social cognitive processes.

The fronto-median wall has been associated in recent studies with subserving a crucial role in social cognitive processes, such as mentalizing and self-referential processing (Amodio \& Frith, 2006). Mentalizing includes meta-cognitive representations needed when attending to our own mental states as well as the mental states of others (Frith \& Frith, 2003). Similarly, in hierarchical models on lateral PFC functions the anterior prefrontal cortex (aPFC) is thought to mediate domain-general, meta-control processes, needed to integrate or resolve occurring conflicts between different cognitive sub-processes (Christoff \& Gabrieli, 2000; Koechlin et al., 2003; Ramnani \& Owen, 2004). Furthermore, the mPFC is associated with non-automatic cognitive processes, which have to be self- 
initiated and -guided, reflecting the intentional aspects of theses processes (Damasio, 1999; Devinsky et al., 1995).

Crucially, sub-processes of these complex functions may be related to the suppression of motor mimicry. Deficits in such a relevant meta-cognitive capacity might also affect the building of representations concerning one's own motor intentions. This suggests that deficits in this metacognitive capacity might affect the building of representations concerning one's own intentions, as well as the attribution of intentions or beliefs to others. Consistent with this view, it was found that in the frontal lesion group impaired mentalizing ability was associated with decreased performance in controlling imitative behaviour, reflecting the impaired building of such abstract representations. Unfortunately, our patient data did not allow a more fine-grained functional-neuroanatomical specification of this relationship. Correlations were found in a group of patients, which had lesions in the medial, as well as in the lateral part of the frontal cortex. In the literatue there is a clear inconsistency between findings on mentalizing from brain imaging and neuropsychology. While neuroimaging results seem to suggest that the fronto-median wall is the critical region in mentalizing tasks, several lesions studies have revealed that also damage to the lateral PFC can cause impairments in ToM tasks (e.g. Griffin et al., 2006; Happe et al., 1999) and that bilateral damage to the mPFC does not necessarily impair mentalizing (Bird et al., 2004). One likely explanation for these findings might be that the aPFC operates as a functional system, which might also depend on the integrity of other prefrontal regions in the lateral prefrontal cortex (Shaw et al., 2005).

Another region which has been found previous neuroimaging studies on the control of imitative behavior and in studies investigating mental and spatial perspective-taking, is the TPJ region (Frith \& Frith, 2003; Samson et al., 2004; Saxe, 2006). This which would suggest again that similar functions are needed in both sets of tasks, such as the ability for differentiation of self- and other-related representations in the mental or spatial domain. This idea is supported by the finding that in the group with lesions around the TPJ region, the ability to inhibit imitative responses was 
correlated with self-reported, cognitive perspective-taking skills and the ability to adopt different visual perspectives.

First, this is consistent with findings on cognitive perspective-taking and sense of agency. A series of studies on motor and mental perspective-taking yielded consistently activations of the TPJ, reflecting the need to distinguish the self and other-person perspective (Ruby \& Decety, 2001, 2003). Furthermore, studies investigating the sense of agency, i.e. the feeling that actions are caused by oneself, and can be distinguished from externally caused events (Gallagher, 2000), have also found activations of the TPJ region (e.g. Farrer \& Frith, 2002; Leube et al., 2003). The ability to adopt another perspective might therefore crucially depend on the realisation that different perspectives exist, with the self-perspective being one possible perspective among other, and the separation of these perspectives (Frith, 2007; Ruby \& Decety, 2003). In line with this view, a recent fMRI study showed that the degree of self-other overlap was negatively correlated with activity in the inferior parietal lobe during a social perception task (Lawrence et al., 2006). Importantly, the behavioural data revealed that less self-other overlap, indexing an increased ability for self-other differentiation, resulted in improved accuracy during the social perception task. This suggests that there is an optimal level of self-other overlap and that a complete blurring of self- and other-perspective might be disadvantageous (Lamm et al., 2007). The capacity for self-other differentiation therefore could be a key component of cognitive perspective-taking and the control of imitative responses. This functional mechanism might underlie behavioural performance in both tasks, perspective-taking and imitative control, by attributing of either thoughts or action related signals to the correct agent (Decety \& Grezes, 2006). Second, recent imaging studies on visual perspective-taking, that is a translocation of an egocentric viewpoint, have also activated the TPJ region (Aichhorn et al., 2006; Zacks, et al., 1999, 2003). It is assumed that the TPJ creates representations of one's body according to inputs from the environment through the integration of multimodal signals. This allows the monitoring of the correspondence of these signals and therefore contributes to the feeling of agency, or self-other 
distinction (Blanke \& Arzy, 2005; Farrer \& Frith, 2002). Similarly, controlling imitative behaviour requires relating one's own body configuration to the observed body movement to assess the selfagency. Thus, underlying this ability and spatial perspective-taking might be a multisensory, bodyrelated mechanism for agency, (spatial) perspectivity and self-unity.

Crucially in this context is that the TPJ region has not only been associated with self-other distinction, which might contribute as a low-level process to the ability to represent and decouple others' mental states (Frith \& Frith, 2003), but it has also been proposed that this site is specifically involved in ToM processing (Samson et al., 2004; Perner et al., 2006; Saxe \& Wexler, 2005). This view would suggest that in the temporo-parietal group the ability to mentalize should also be correlated with the inhibition of imitation. Although no significant correlation was found between these two measures in the first analysis, a closer inspection of the data revealed that this correlation was concealed by three outliers, which showed lesions only to the left TPJ area and were also found to have mild to medium impairments in language comprehension in a previous neuropsychological assessment. When excluding those outliers, a negative correlation (imitation-inhibition task (RTs) and Tom accuracy score, $r(9)=-.55, \mathrm{p}=.09$, mental state verbs, $r(9)=-.50, \mathrm{p}=.013$, uncorrected) evolved. It might be therefore possible that the decreased performance of those patients was in fact caused by language comprehension deficits which might have affected the ToM task and the imitation-inhibition task unproportionally.

\section{Using an Associative Approach to Investigate Cognitive Functions}

One potential methodological caveat of the present study might be that we aimed at finding associations between tasks rather then dissociations. Looking for dissociations rather than associations is the more common procedure in neuropsychological studies (Shallice, 1988). Artificial associations between tasks may arise by a lesion affecting two functional systems, which are localised in close proximity to each other in the brain. Although, the present study only included patients with focal lesions, confined to one lobe, it is still possible that two functional regions were 
affected by these lesions. Nevertheless, an associative approach is justified in the present case, because the hypothesis for this patient study was derived from brain imaging data that suggested a functional overlap of the inhibition of imitative behaviour and social cognitive skills. Furthermore, we provided additional empirical data that is not consistent with the idea that the correlation in the patient group might be due to a lesion overlap in non-relevant brain areas. It was tested whether the general pattern of results in the patient group can be replicated in the control group. In principle, the hypothesis of shared sub-processes between tasks holds also true for a normal population, as it makes general assumptions on underlying elementary mechanisms. Therefore, the same hypothesis can be investigated with a normal sample, but is hampered by the fact that the variation in this group might be too small to find reliable associations between tasks. However, if in a normal population the same association can be found as in a lesion group, this association then cannot rely on concurrent damage to two functional systems, but is likely to occur, because two tasks share common functions. Similar to the frontal group, the control group in the present study also showed a significant correlation between the performance of the ToM task and the imitation-inhibition task, although, as expected, to a lesser extent. Furthermore, the influence of possible moderator variables was carefully controlled.

\section{Shared Motoric Representations and Higher-Level Social Cognition: an Integrative Approach}

In conclusion, our findings strengthen the idea, derived from neuroimaging, that controlling automatic, motor representations draws onto key sub-processes needed in higher-level social cognition (Spengler et al., 2009). Recently, it has been argued recently that shared motoric representations underlie action understanding and social cognition (Rizzolatti \& Craighero, 2004). Although this mirror-matching mechanism may be an underlying part of empathy and mentalizing, it might provide only an essential basis for more advanced mind-reading abilities (Frith \& Frith, 2006b). The emergence of mature mind-reading abilities may rely on the ability to build up different representations of self and other and to distinguish those, needed, for example, when attributing mental states which are different from own beliefs in the false belief task or distinguishing between 
being imitated from imitating another person (Chaminade et al., 2005; Hurley, 2005). These specific mechanisms are thus rather related to the control of shared representations than to sharing representations per se (Brass \& Spengler, 2008; Brass et al., 2009; Decety \& Grezes, 2006; Frith \& Frith, 2006b). Consequently, this functional description of the cognitive processes implicated in mentalizing overlaps with our conceptualization of the inhibition of mimicry behaviour. The inhibition of imitative behaviour can be understood as a prime example for assigning agency and exerting control over the shared representational system. Constitutive functional mechanisms, developed to distinguish self and other in the motor domain, might have subsequently generalized to more abstract representations such as mental states and might thus be conceptualised as elementary precursors for more evolved social cognitive functions (Brass et al., 2005). In this sense, our findings allow to integrate two hitherto mutually exclusive positions on the origin of mind reading, by linking shared representations and mentalizing through the mechanisms involved in the control of shared representations. This interpretation allows a novel integrative view on embodied and social cognitive functions (see also Keysers \& Gazzola, 2006) and opens up new perspectives for the investigation and conceptualization of these functions during development and adulthood. 


\section{References}

Aichhorn, M., Perner, J., Kronbichler, M., Staffen, W., \& Ladurner, G. (2006). Do visual perspective tasks need theory of mind? NeuroImage, 30(3), 1059-1068.

Amodio, D. M., \& Frith, C. D. (2006). Meeting of minds: the medial frontal cortex and social cognition. Nature Reviews Neuroscience, 7(4), 268-277.

Baird, A., Dewar, B. K., Critchley, H., Gilbert, S. J., Dolan, R. J., \& Cipolotti, L. (2006). Cognitive functioning after medial frontal lobe damage including the anterior cingulate cortex: a preliminary investigation. Brain and Cognition, 60(2), 166-175.

Barbas, H., Ghashghaei, H., Dombrowski, S. M., \& Rempel-Clower, N. L. (1999). Medial prefrontal cortices are unified by common connections with superior temporal cortices and distinguished by input from memory-related areas in the rhesus monkey. Journal of Comparative Neurology, 410(3), 343-367.

Barresi, J., Moore, C. (1996). Intentional relations and social understanding. Behavioural and Brain Sciences, 19, 107-154.

Blanke, O., \& Arzy, S. (2005). The out-of-body experience: disturbed self-processing at the temporo-parietal junction. Neuroscientist, 11(1), 16-24.

Bird, C. M., Castelli, F., Malik, O., Frith, U., \& Husain, M. (2004). The impact of extensive medial frontal lobe damage on 'Theory of Mind' and cognition. Brain, 127(Pt 4), 914-928.

Bowden, S. C., Fowler, K.S., Bell, R.C., Whelan, G., Clifford, C.C., Ritter, A.J., Long, C.M. (1998). The reliability and internal validity of the Wisconsin Card Sorting Test. Neuropsychological Rehabilitation, 8, 243-254.

Brass, M., Spengler, S. (2008). The inhibition of imitative behavior and attribution of mental states. In T. Striano, Reid, V. (Ed.), Social Cognition: Development, Neuroscience and Autism. Oxford: Blackwell.

Brass, M., Bekkering, H., \& Prinz, W. (2001a). Movement observation affects movement 
execution in a simple response task. Acta Psychologica (Amst), 106(1-2), 3-22.

Brass, M., Bekkering, H., Wohlschlager, A., \& Prinz, W. (2000). Compatibility between observed and executed finger movements: comparing symbolic, spatial, and imitative cues. Brain and Cognition, 44(2), 124-143.

Brass, M., Derrfuss, J., Matthes-von Cramon, G., \& von Cramon, D. Y. (2003). Imitative response tendencies in patients with frontal brain lesions. Neuropsychology, 17(2), 265-271.

Brass, M., Derrfuss, J., \& von Cramon, D. Y. (2005). The inhibition of imitative and overlearned responses: a functional double dissociation. Neuropsychologia, 43(1), 89-98.

Brass, M., \& Heyes, C. (2005). Imitation: is cognitive neuroscience solving the correspondence problem? Trends in Cognitive Sciences, 9(10), 489-495.

Brass, M., Ruby, P. \& Spengler, S. (2009). Inhibition of imitative behavior and social cognition. Philosophical Transactions of the Royal Society of London, 364, 2359-2367.

Brass, M., Zysset, S., \& von Cramon, D. Y. (2001b). The inhibition of imitative response tendencies. Neuroimage, 14(6), 1416-1423.

Chaminade, T., Meltzoff, A. N., \& Decety, J. (2005). An fMRI study of imitation: action representation and body schema. Neuropsychologia, 43(1), 115-127.

Chartrand, T. L., \& Bargh, J. A. (1999). The chameleon effect: the perception-behavior link and social interaction. Journal of Personality and Social Psychology, 76(6), 893-910.

Christoff, K. \& Gabrieli, J.D.E. (2000). The frontopolar cortex and human cognition: Evidence for a rostrocaudal hierarchical organization with the human prefrontal cortex. Psychobiology, 28(2), 168-186.

Damasio, A. R. (1999). The feeling of what happens: Body and emotion in the making of consciousness. New York: Harcourt Brace.

Davis, M. (1980). A multidimensional approach to individual differences in empathy. JSAS Catalog of Selected Documents in Psychology, 10, 85. 
Davis, M. (1983). Measuring individual differences in empathy: Evidence for a multidimensional approach. Journal of Personality and Social Psychology, 44, 113-126.

Decety, J., Chaminade, T., Grezes, J., \& Meltzoff, A. N. (2002). A PET exploration of the neural mechanisms involved in reciprocal imitation. Neuroimage, 15(1), 265-272.

Decety, J., \& Grezes, J. (2006). The power of simulation: imagining one's own and other's behavior. Brain Research, 1079(1), 4-14.

Decety, J., \& Lamm, C. (2007). The role of the right temporoparietal junction in social interaction: how low-level computational processes contribute to meta-cognition. Neuroscientist, 13(6), 580-593.

Devinsky, O., Morrell, M. J., \& Vogt, B. A. (1995). Contributions of anterior cingulate cortex to behaviour. Brain, 118 ( Pt 1), 279-306.

Enz, S., Zoll, C., Schaub, H. (2004). Validierung eines Fragebogens zur Messung von Empathie. In T. Ramsayer, Grabianowski, S., Troche, S. (Ed.), 44. Kongress der Deutschen Gesellschaft für Psychologie. Lengerich: Pabst.

Farrer, C., \& Frith, C. D. (2002). Experiencing oneself vs another person as being the cause of an action: the neural correlates of the experience of agency. Neuroimage, 15(3), 596-603.

Fellows, L. K., \& Farah, M. J. (2005). Is anterior cingulate cortex necessary for cognitive control? Brain, 128(Pt 4), 788-796.

Frith, C. D. (2007). The social brain? Philosophical Transactions of the Royal Society of London, 362(1480), 671-678.

Frith, C. D., \& Frith, U. (2006a). How we predict what other people are going to do. Brain Research, 1079(1), 36-46.

Frith, C. D., \& Frith, U. (2006b). The neural basis of mentalizing. Neuron, 50(4), 531-534.

Frith, U., \& Frith, C. D. (2003). Development and neurophysiology of mentalizing. Philosophical Transactions of the Royal Society of London, 358(1431), 459-473. 
Gallagher, H. L., Happe, F., Brunswick, N., Fletcher, P. C., Frith, U., \& Frith, C. D. (2000). Reading the mind in cartoons and stories: an fMRI study of 'theory of mind' in verbal and nonverbal tasks. Neuropsychologia, 38(1), 11-21.

Gallagher, I. I. (2000). Philosophical conceptions of the self: implications for cognitive science. Trends in Cognitive Sciences, 4(1), 14-21.

Gallese, V., Goldman, A. (1998). Mirror neurons and the simulation theory of mind-reading. Trends in Cognitive Sciences, 2, 493-501.

Gilbert, S. J., Spengler, S., Simons, J. S., Steele, J. D., Lawrie, S. M., Frith, C. D., et al. (2006). Functional specialization within rostral prefrontal cortex (area 10): a meta-analysis. Journal of Cognitive Neuroscience, 18(6), 932-948.

Grant, D. A., Berg, E.A. (1948). A behavioral analysis of degree of reinforcement and ease of shifting to new responses in a Weigl-type card-sorting problem. Journal of Experimental Psychology, 38, 404-411.

Griffin, R., Friedman, O., Ween, J., Winner, E., Happe, F., \& Brownell, H. (2006). Theory of mind and the right cerebral hemisphere: refining the scope of impairment. Laterality, 11(3), 195-225.

Happe, F., Brownell, H., \& Winner, E. (1999). Acquired 'theory of mind' impairments following stroke. Cognition, 70(3), 211-240.

Horn, W. (1983). Leistungsprüfsystem (LPS). Göttingen: Verlag für Psychologie.

Iacoboni, M., Woods, R. P., Brass, M., Bekkering, H., Mazziotta, J. C., \& Rizzolatti, G. (1999). Cortical mechanisms of human imitation. Science, 286(5449), 2526-2528.

Jacobs, B., Schall, M., Prather, M., Kapler, E., Driscoll, L., Baca, S., et al. (2001). Regional dendritic and spine variation in human cerebral cortex: a quantitative golgi study. Cerebral Cortex, $11(6), 558-571$.

Jeannerod, M. (1999). The 25th Bartlett Lecture. To act or not to act: perspectives on the representation of actions. The Quarterly Journal of Experimental Psychology. A, 52(1), 1-29. 
Keysers, C., \& Gazzola, V. (2006). Towards a unifying neural theory of social cognition. Progress in Brain Research, 156, 379-401.

Kilner, J. M., Paulignan, Y., \& Blakemore, S. J. (2003). An interference effect of observed biological movement on action. Current Biology, 13(6), 522-525.

Koechlin, E., Ody, C. \& Kouneiher, F. (2003). The architecture of cognitive control in the human prefrontal cortex. Science, 302(5648), 1181-1185.

Lamm, C., Batson, C. D., \& Decety, J. (2007). The neural substrate of human empathy: effects of perspective-taking and cognitive appraisal. Journal of Cognitive Neuroscience, 19(1), 42-58.

Lawrence, E. J., Shaw, P., Giampietro, V. P., Surguladze, S., Brammer, M. J., \& David, A. S. (2006). The role of 'shared representations' in social perception and empathy: an fMRI study. Neuroimage, 29(4), 1173-1184.

Lehrl, S. (1999). Mehrfach-Wortschatz-Intelligenztest (MWT-B). Erlangen: Straube.

Leube, D. T., Knoblich, G., Erb, M., Grodd, W., Bartels, M., \& Kircher, T. T. (2003). The neural correlates of perceiving one's own movements. Neuroimage, 20(4), 2084-2090.

Lhermitte, F., Pillon, B., Serdaru, M. (1986). Human autonomy and the frontal lobes. Part I: Imitation and utilization behavior: a neuropsychological study of 75 patients. Annals of Neuology, 19(4), 326-334.

Nelson, H. E. (1976). A modified card sorting test sensitive to frontal lobe defects. Cortex, 12, 313-324.

Nelson, H. E., Willison, J. (1991). National Adult Reading Test (NART): Test manual. Windsor: NFER-Nelson.

Northoff, G., \& Bermpohl, F. (2004). Cortical midline structures and the self. Trends in Cognitive Sciences, 8(3), 102-107.

Ochsner, K. N., Knierim, K., Ludlow, D. H., Hanelin, J., Ramachandran, T., Glover, G., et al. (2004). Reflecting upon feelings: an fMRI study of neural systems supporting the attribution of 
emotion to self and other. Journal of Cognitive Neuroscience, 16(10), 1746-1772.

Parsons, L. M. (1987). Imagined spatial transformation of one's body. Journal of Experimental Psychology: General, 116, 172-191.

Paulus, C. (2004). Saarbrücker Persönlichkeits-Fragebogen. Retrieved August, 15, 2005, from www.uni-saarland.de/fak5/ezw/personal/paulus/empathy/SPF(IRI)_V3.1a.pdf

Perner, J., Aichhorn, M., Kronbichler, M., Staffen, W., Ladurer, G. (2006). Thinking of mental and other representations: The roles of the left and right temporo-parietal junction. Social Neuroscience, 1, 245-258.

Prinz, W. (1997). Perception and action planning. European Journal of Cognitive Psychology, 9, 129-154.

Ramnani, N., \& Owen, A. M. (2004). Anterior prefrontal cortex: insights into function from anatomy and neuroimaging. Nature Reviews Neuroscience, 5(3), 184-194.

Rizzolatti, G., \& Craighero, L. (2004). The mirror-neuron system. Annual Review of Neuroscience, 27, 169-192.

Ruby, P., \& Decety, J. (2001). Effect of subjective perspective taking during simulation of action: a PET investigation of agency. Nature Neuroscience, 4(5), 546-550.

Ruby, P., \& Decety, J. (2003). What you believe versus what you think they believe: a neuroimaging study of conceptual perspective-taking. The European Journal of Neuroscience, 17(11), 2475-2480.

Samson, D., Apperly, I. A., Chiavarino, C., \& Humphreys, G. W. (2004). Left temporoparietal junction is necessary for representing someone else's belief. Nature Neuroscience, 7(5), 499-500.

Saxe, R. (2006). Uniquely human social cognition. Current Opinion in Neurobiology, 16(2), 235-239.

Saxe, R., \& Wexler, A. (2005). Making sense of another mind: the role of the right temporoparietal junction. Neuropsychologia, 43(10), 1391-1399. 
Shallice, T. (1988). From neuropsychology to mental structure. Cambridge: University Press.

Spengler, S., von Cramon, D.Y. \& Brass, M. (2009). Control of shared representations relies on key processes involved in mental state attribution. Human Brain Mapping, doi: 10.1002/hbm.20800.

Shaw, P., Bramham, J., Lawrence, E. J., Morris, R., Baron-Cohen, S., \& David, A. S. (2005). Differential effects of lesions of the amygdala and prefrontal cortex on recognizing facial expressions of complex emotions. Journal of Cognitive Neuroscience, 17(9), 1410-1419.

Swick, D., \& Turken, A. U. (2002). Dissociation between conflict detection and error monitoring in the human anterior cingulate cortex. Proceedings of the National Academy of Sciences of the United States of America, 99(25), 16354-16359.

Vogeley, K., Bussfeld, P., Newen, A., Herrmann, S., Happe, F., Falkai, P., et al. (2001). Mind reading: neural mechanisms of theory of mind and self-perspective. Neuroimage, 14(1), 170-181.

Wechsler, D. (1987). Wechsler Memory Scale- Revised. San Antonio: Hartcourt Brace.

Zacks, J., Rypma, B., Gabrieli, J. D., Tversky, B., \& Glover, G. H. (1999). Imagined transformations of bodies: an fMRI investigation. Neuropsychologia, 37(9), 1029-1040.

Zacks, J. M., Tversky, B. (2005). Multiple systems for spatial imagery: Transformations of objects and bodies. Spatial Cognition and Computation, 5(4), 271-306.

Zacks, J. M., Ollinger, J. M., Sheridan, M. A., \& Tversky, B. (2002). A parametric study of mental spatial transformations of bodies. Neuroimage, 16(4), 857-872. 


\section{Appendix}

Lesion description and etiology of the patient groups

\begin{tabular}{|c|c|c|c|c|}
\hline Patient & Group & Lesion Site & Side & Etiology \\
\hline 1 & frontal & $\begin{array}{l}\text { inferior frontal gyrus, } \\
\text { medial frontal gyrus, } \\
\text { anterior insula }\end{array}$ & $\bar{R}$ & stroke \\
\hline 2 & frontal & Frontal pole & $\mathrm{Bi}(1)$ & TBI \\
\hline 3 & frontal & Precentral gyrus & $\mathrm{L}$ & stroke \\
\hline 4 & frontal & $\begin{array}{l}\text { Inferior frontal gyrus, } \\
\text { anterior mid-frontal cortex } \\
\text { fusiform gyrus }\end{array}$ & $\mathrm{Bi}(\mathrm{r})$ & TBI \\
\hline 5 & frontal & precentral sulcus & $\mathrm{L}$ & stroke \\
\hline 6 & frontal & fronto-orbital, fronto-polar & $\mathrm{Bi}$ & TBI \\
\hline 7 & frontal & $\begin{array}{l}\text { fronto-orbital, } \\
\text { fronto-polar }\end{array}$ & $\mathrm{Bi}$ & TBI \\
\hline 8 & frontal & inferior frontal gyrus & $\mathrm{L}$ & $\begin{array}{l}\text { intra-cerebral } \\
\text { bleeding (ICB) }\end{array}$ \\
\hline 9 & frontal & $\begin{array}{l}\text { fronto-orbital, superior } \\
\text { frontal gyrus }\end{array}$ & $\mathrm{Bi}$ & TBI \\
\hline 10 & frontal & $\begin{array}{l}\text { fronto-orbital (lateral), } \\
\text { inferior frontal gyrus, } \\
\text { anterior insula, } \\
\text { putamen }\end{array}$ & $\mathrm{L}$ & stroke \\
\hline 11 & frontal & inferior precentral & $\mathrm{L}$ & stroke \\
\hline
\end{tabular}


sulcus,fusiform gyrus,

lingual gyrus

12

frontal

fronto-orbital (lateral),

L

stroke

inferior frontal gyrus,

precentral gyrus, anterior

insula

13

frontal

fronto-orbital (medial),

$\mathrm{Bi}$

TBI

fronto-polar

14

frontal

fronto-orbital (medial),

$\mathrm{Bi}$

TBI

fronto-polar

15

frontal

fronto-orbital (medial),

L

stroke

fronto-polar, nucleus

caudatus

16

temp-par

Inferior temporal gyrus,

$\mathrm{R}$

encephalitis

angular gyrus,

supramarginal gyrus

17

temp-par

temporo-polar, superior/

$\mathrm{L}$

TBI, stroke

middle temporal gyrus,

angular gyrus, lateral

occipital

18

temp-par

superior/middle temporal

$\mathrm{L}$

stroke

gyrus, angular gyrus,

supramarginal gyrus,

intraparietal sulcus, lateral/

medial occipital 
postcentral gyrus,

L

stroke

supramarginal gyrus,

intraparietal sulcus, superior

temporal gyrus (posterior)

temporal pole, superior

$\mathrm{R}$

stroke

temporal gyrus (posterior),

posterior insula,

supramarginal gyrus

superior temporal gyrus

L tumor

(posterior), supramarginal

gyrus

superior temporal gyrus,

R

stroke

supramarginal gyrus

posterior insula

superior/middle temporal

$\mathrm{R}$

stroke

gyrus, supramarginal gyrus

posterior insula

superior/middle temporal

$\mathrm{R}$

stroke

gyrus, supramarginal gyrus,

angular gyrus, posterior

insula

25

temp-par

superior/middle temporal

$\mathrm{R}$

stroke

gyrus, supramarginal gyrus

posterior insula

L

stroke 
sulcus (posterior), angular

gyrus

27

temp-par

superior temporal gyrus/

L

stroke

sulcus (posterior), angular

gyrus

28

temp-par

superior parietal lobe (R),

$\mathrm{Bi}$

stroke

angular gyrus (L),

supramarginal gyrus (L) 


\section{Footnotes}

${ }^{1}$ The Bonferroni correction of the alpha niveau was computed by dividing the alpha level (one-tailed) by the number of comparisons, separately for each group and task. This resulted in an adjusted alpha level of 0.0166 (for $\mathrm{p}<0.05$ ) and 0.0083 (for $\mathrm{p}<0.01$ ) for the ToM task, and an alpha level of 0.0125 (for $\mathrm{p}<0.05$ ) and 0.0062 (for $\mathrm{p}<0.01$ ) for the other tasks. 
Table 1

Demographic data of the sample

\begin{tabular}{llll}
\hline & $\begin{array}{l}\text { Frontal lesion } \\
\text { group }\end{array}$ & $\begin{array}{l}\text { TPJ lesion group } \\
(\mathbf{n}=\mathbf{1 5})\end{array}$ & $\begin{array}{l}\text { Healthy controls } \\
(\mathbf{n}=\mathbf{2 8})\end{array}$ \\
& $10 / 5$ & & \\
\hline Gender $(\mathrm{m} / \mathrm{f})^{\mathrm{a}}$ & $48.53(10.57)$ & $53.07(6.67)$ & $50.05(9.06)$ \\
Age (years) & & $11 / 2$ & $12.50(2.49)$ \\
Education $^{\mathrm{a}}$ & $11.80(2.21)$ & $12.54(2.44)$ & -- \\
Etiology $^{\mathrm{b}}$ & $8 / 7 / 0 / 0$ & $10 / 1 / 1 / 1$ & $101.75(18.52)$ \\
IQ $^{\mathrm{a}, \mathrm{c}}$ & $100.00(13.06)$ & $105.66(17.84)$ & \\
\hline
\end{tabular}

Note: ${ }^{\mathrm{a}}$ Mean (SD). ${ }^{\mathrm{b}}$ vascular etiology/ TBI/ tumor/ infection. ${ }^{\mathrm{c}}$ Mehrfachwortschatztest, NART equivalent 
Table 2

Correlations between the variables of the imitation-inhibition task and the Theory of Mind task

\begin{tabular}{lllll}
\hline & \multicolumn{2}{l}{ frontal lesion group } & temporo-parietal lesion group \\
\hline & \multicolumn{2}{l}{ Imitation-inhibition } & Imitation-inhibition \\
& \multicolumn{2}{l}{ Interference score } & Interference score \\
\cline { 2 - 5 } & $\mathrm{RT}$ & $\mathrm{RT}$ & errors \\
\hline ToM condition- & $\mathbf{r}=\mathbf{- . 7 5 7 * *}$ & $\mathrm{r}=-.006$ & $\mathrm{r}=-.023$ & $\mathrm{r}=.063$ \\
accuracy score & $\mathbf{p}=\mathbf{. 0 0 1}$ & $\mathrm{p}=.984$ & $\mathrm{p}=.941$ & $\mathrm{p}=.837$ \\
\hline physical & $\mathbf{r}=\mathbf{- . 5 5 1}$ & $\mathrm{r}=-.171$ & $\mathrm{r}=-.358$ & $\mathrm{r}=-.378$ \\
condition- & $\mathbf{p}=\mathbf{. 0 3 3}$ & $\mathrm{p}=.543$ & $\mathrm{p}=.230$ & $\mathrm{p}=.203$ \\
accuracy score & & & & $\mathrm{r}=-.231$ \\
\hline ToM condition- & $\mathbf{r}=\mathbf{- . 6 2 8 *}$ & $\mathrm{r}=-.398$ & $\mathrm{r}=-.205$ \\
mental state verbs & $\mathbf{p}=\mathbf{. 0 1 2}$ & $\mathrm{p}=.142$ & $\mathrm{p}=.448$ & $\mathrm{p}=.501$ \\
\hline
\end{tabular}

Note: Spearman's rho correlations for the frontal lesion group $(\mathrm{N}=15)$ and the temporo-parietal lesion group $(\mathrm{N}=13)$. P-values are uncorrected, two-tailed values. Dark grey cells are statistically significant on the corrected Bonferroni alpha level (one-tailed), $* *$ indicate $\mathrm{p}<0.01 / *$ indicates $\mathrm{p}<$ 0.05 , light grey cells show a tendency towards statistical significance $(\mathrm{p}<0.05$, uncorrected $)$. 
Table 3

Correlations between the variables of the imitation-inhibition task and the empathy subscales

\begin{tabular}{|c|c|c|c|c|}
\hline & \multicolumn{2}{|c|}{ frontal lesion group } & \multicolumn{2}{|c|}{ temporo-parietal lesion group } \\
\hline & \multicolumn{2}{|c|}{ Imitation-inhibition } & \multicolumn{2}{|c|}{ Imitation-inhibition } \\
\hline & \multicolumn{2}{|c|}{ Interference score } & \multicolumn{2}{|c|}{ Interference score } \\
\hline & RT & errors & RT & errors \\
\hline cognitive & $r=-.329$ & $\mathrm{r}=.072$ & $\mathbf{r}=-.775 * *$ & $r=-.555$ \\
\hline perspective-taking & $\mathrm{p}=.231$ & $\mathrm{p}=.797$ & $p=.002$ & $p=.049$ \\
\hline \multirow[t]{2}{*}{ fantasy } & $\mathrm{r}=-.168$ & $\mathrm{r}=-.271$ & $\mathrm{r}=-.246$ & $r=-.122$ \\
\hline & $\mathrm{p}=.551$ & $\mathrm{p}=.328$ & $\mathrm{p}=.417$ & $p=.692$ \\
\hline \multirow[t]{2}{*}{ empathic concern } & $r=-.223$ & $r=-.174$ & $r=-.424$ & $r=-.428$ \\
\hline & $\mathrm{p}=.424$ & $\mathrm{p}=.535$ & $\mathrm{p}=.149$ & $\mathrm{p}=.145$ \\
\hline ideomotor & $\mathrm{r}=.009$ & $\mathrm{r}=-.161$ & $r=-.742$ & $r=-.335$ \\
\hline empathy & $\mathrm{p}=.979$ & $p=.636$ & $p=.014$ & $\mathrm{p}=.343$ \\
\hline
\end{tabular}

Note: Spearman's rho correlations for the frontal lesion group (IRI subscales $\mathrm{N}=15$, ideomotor subscale $\mathrm{N}=11$ ) and the temporo-parietal lesion group (IRI subscales $\mathrm{N}=13$, ideomotor subscale $\mathrm{N}=$ 10). P-values are uncorrected, two-tailed values. Dark grey cells are statistically significant on the corrected Bonferroni alpha level (one-tailed), ** indicate $\mathrm{p}<0.01 / *$ indicates $\mathrm{p}<0.05$, light grey cells show a tendency towards statistical significance ( $p<0.05$, uncorrected). 
Table 4

Correlations between the variables of the imitation-inhibition task and the visual perspective-taking task

\begin{tabular}{lllll}
\hline & \multicolumn{2}{l}{ frontal lesion group } & temporo-parietal lesion group \\
& \multicolumn{2}{l}{ Imitation-inhibition } & Imitation-inhibition \\
& \multicolumn{2}{l}{ Interference score } & Interference score \\
\cline { 2 - 5 } & $\mathrm{RT}$ & errors & $\mathrm{RT}$ & errors \\
\hline Hand condition- & $\mathrm{r}=.071$ & $\mathrm{r}=.107$ & $\mathrm{r}=.413$ & $\mathrm{r}=-.012$ \\
reaction times & $\mathrm{p}=.817$ & $\mathrm{p}=.728$ & $\mathrm{p}=.235$ & $\mathrm{p}=.973$ \\
\hline Side condition- & $\mathrm{r}=.214$ & $\mathrm{r}=.127$ & $\mathrm{r}=.678$ & $\mathrm{r}=-.188$ \\
reaction times & $\mathrm{p}=.428$ & $\mathrm{p}=.680$ & $\mathrm{p}=.045$ & $\mathrm{p}=.628$ \\
\hline Hand condition- & $\mathrm{r}=.213$ & $\mathrm{r}=.-.145$ & $\mathrm{r}=.758 *$ & $\mathrm{r}=.196$ \\
errors & $\mathrm{p}=.484$ & $\mathrm{p}=.637$ & $\mathrm{p}=.011$ & $\mathrm{p}=.587$ \\
\hline Side condition- & $\mathrm{r}=.009$ & $\mathrm{r}=.126$ & $\mathrm{r}=-.023$ & $\mathrm{r}=-.293$ \\
errors & $\mathrm{p}=.976$ & $\mathrm{p}=.682$ & $\mathrm{p}=.953$ & $\mathrm{p}=.445$ \\
\hline
\end{tabular}

Note: Spearman's rho correlations for the frontal lesion group $(\mathrm{n}=13)$ and the temporo-parietal lesion group (hand condition $n=10$, side condition $n=9$, after elimination of one outlier). Errors include errors and omissions. P-values are uncorrected, two-tailed values. Dark grey cells are statistically significant on the corrected Bonferroni alpha level (one-tailed), $* *$ indicate $\mathrm{p}<0.01 / *$ indicates $\mathrm{p}<0.05$, light grey cells show a tendency towards statistical significance $(\mathrm{p}<0.05$, uncorrected). 


\section{Figure Captions}

Figure 1. Imitation-inhibition task. Displayed are example pictures for the incongruent, congruent and baseline condition (from left to right).

Figure 2. Scatter plots of the significant correlations in the patient groups between the performance in the imitation-inhibition task and the social cognitive tasks.

Top: frontal lesion group, $\mathrm{N}=15$, bottom: temporo-parietal group, $\mathrm{N}=13, \mathrm{~N}=10$ for the visual perspective-taking task

Figure 3. Scatter plots of the correlations between imitative control and mentalizing

Left: Scatter plot showing the significant correlation in the frontal lesions group $(\mathrm{N}=15)$ between the performance in the imitation-inhibition task and the accuracy score (ToM condition), after statistically eliminating the performance in the physical condition. Right: Scatter plot displaying the significant correlation in the control group $(\mathrm{N}=27)$ between the performance in the imitationinhibition task and the accuracy score (ToM condition), after statistically eliminating the performance in the physical condition. 


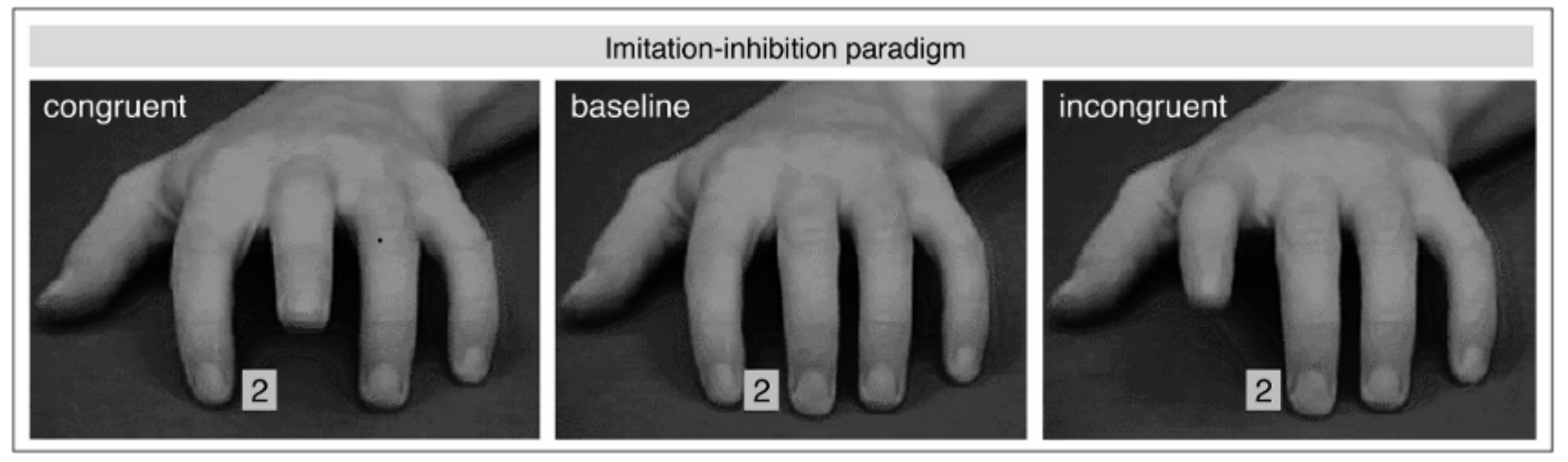




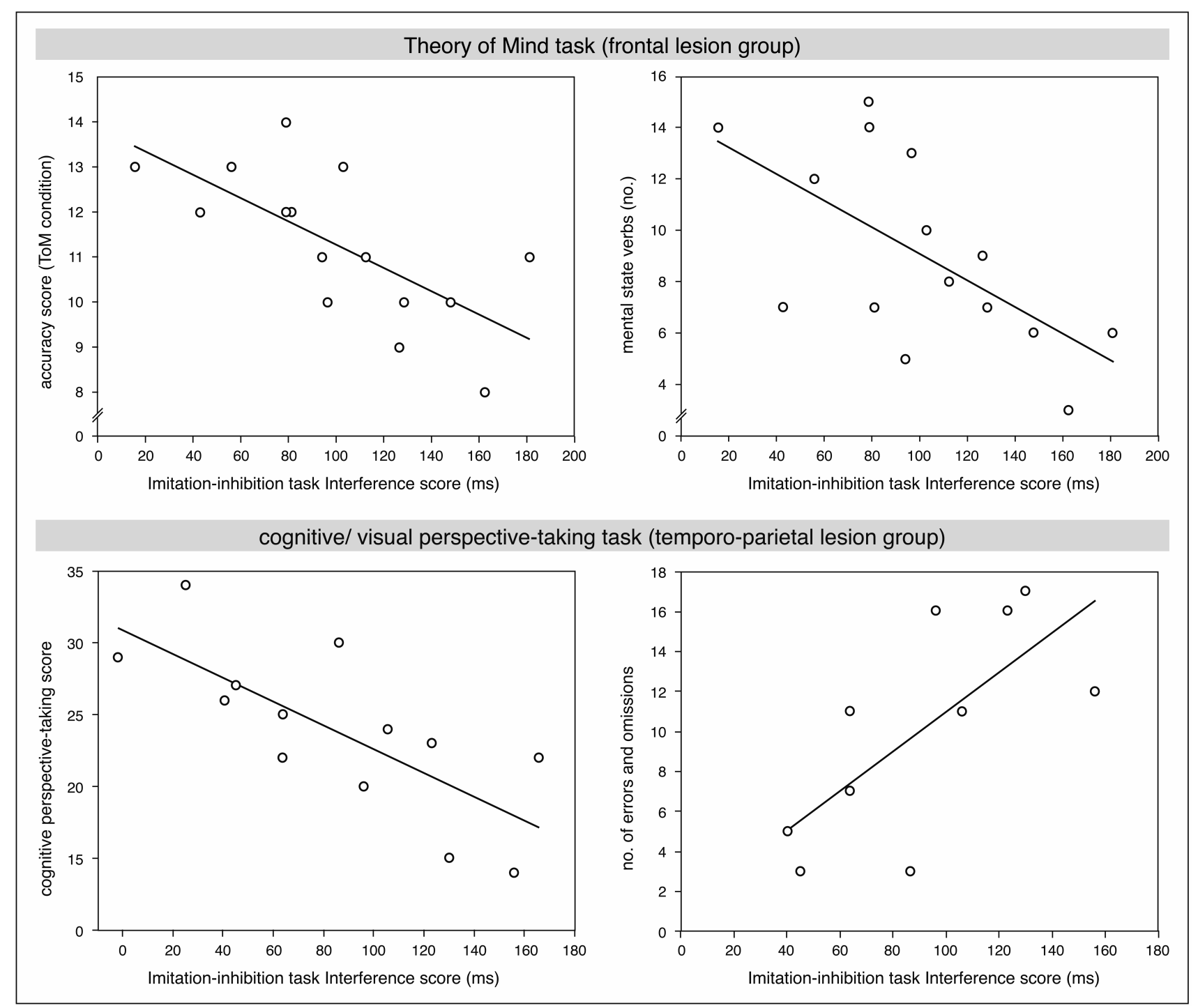

42 


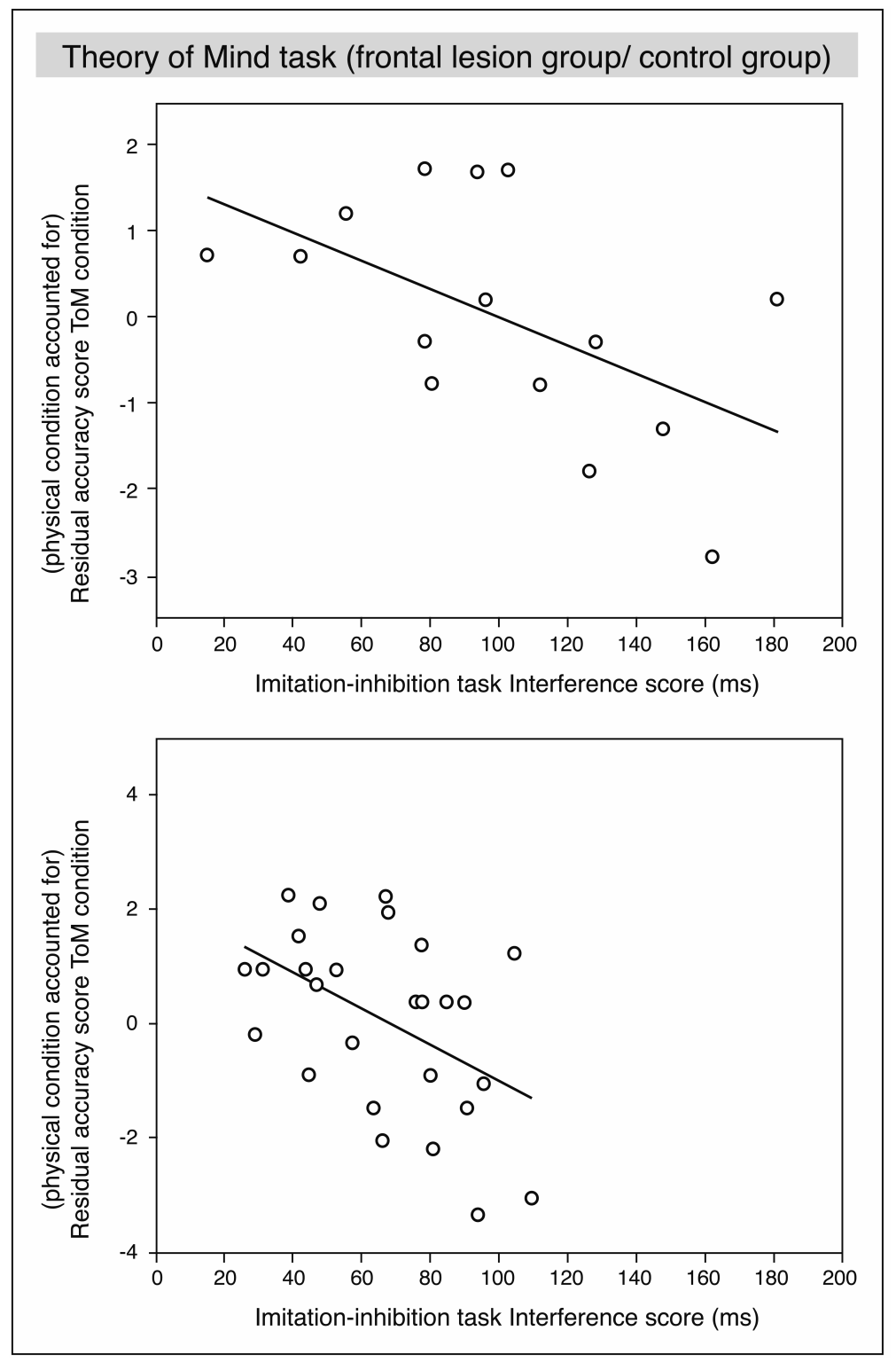

